\title{
New tool for Swiss army knife
}

\section{Ortrun Mittelsten Scheid}

ife as a virus is demanding. Tiny in size, and with limited genetic material, a -virus must successfully take over comparatively gigantic cells or multicellular organisms. Unlike symbiotic invaders it will face a hostile reception, and it is no surprise that viruses have evolved to carry simplified, multifunctional conquest weapons, resembling the sophisticated equipment in the pocket of 007's dinner suit. A good example of such a weapon is the 'helper component protease' (HC-Pro), which is found in the group of potyviruses that infect, and cause severe disease in, a spectrum of plant species. Three papers published in Proceedings of the National Academy of Sciences ${ }^{1}$, EMBO Journal ${ }^{2}$ and $\mathrm{Cell}^{3}$ now tell us something about why HCPro makes these viruses so successful.

As indicated by its name, HC-Pro was identified as the protease that cleaves a viral polyprotein into its functional subunits. But, like a Swiss army knife, it packs in more than just a cutting blade. It binds RNA and is involved in amplification of the viral RNA genome, spreading the virus from the site of infection and transmitting it from plant to plant via sap-sucking insects ${ }^{4}$. Although almost all plant viruses have proteins to do these jobs (albeit in different combinations), the new work ${ }^{1-3}$ shows that HC-Pro has another function unknown in any other virus - it paralyses an important plant defence mechanism that normally acts against viruses.

The mechanism in question is based on 'post-transcriptional gene silencing' (PTGS), whereby specific transcripts are degraded before they can be translated ${ }^{5}$. The process was initially observed in transgenic plants when, instead of being overexpressed, transgenes (as well as homologous endogenous genes) became silent. Cytoplasmic viral templates can be degraded by the same mechanism, especially if parts of the virus have been integrated into the plant's nucleus as transgenes. Indeed, silencing of viral gene expression by PTGS can explain 'recovery' of virusinfected plants which, despite initial viral replication and spread throughout the plant, develop new, virus-free shoots and leaves ${ }^{6}$.

Because RNA viruses replicate through double-stranded RNA intermediates, and silenced transgenes are suspected to produce aberrant antisense RNA, double-stranded RNA could be the common signal for specific degradation, probably also related to the mechanism that operates in animal models ${ }^{7}$. PTGS is thought to have evolved either as an antiviral defence mechanism, or as a regulatory mechanism for endogenous plant genes that also affects viral genes ${ }^{8}$. In both cases, the potential to act against many viral sequences, combined with the high specificity of the response, seems to make PTGS part of a plant's 'immune system' based on particular RNA sequences. Therefore, it is not surprising that, like their animal counterparts, some plant viruses have developed strategies to attack this defence. The HC-Pro protein of tobacco etch virus and protein $2 \mathrm{~b}$ of cucumber mosaic virus are part of this attack. Anandalakshmi et al. ${ }^{1}$, Brigneti et al. ${ }^{2}$ and Kasschau and Carrington ${ }^{3}$ now show that when these proteins are expressed in tobacco (Nicotiana tabacum) and $N$. benthamiana, the PTGS response is suppressed - existing silencing and virus resistance are removed, and silencing cannot be established during viral infection.

These results also bear on the concept of synergistic viral infection. When plants are infected with two different species of virus, the result is often more severe symptoms than inoculation with either virus alone. Potyviruses are successful partners in this double-attack strategy, allowing strong replication and spread of the other partner. HC-Pro is essential for double infection ${ }^{9}$, and it is probably its ability to suppress PTGS that limits the general plant defence response, allowing other viruses that do not encode HC-Pro to be amplified. Viral modulation of the plants' gene-silencing system might also cause unexpected changes in gene expression if transgenic plants cannot be saved from uncontrolled infections.

We do not yet know how, at the molecular level, HC-Pro is involved in viral amplification, spread and transmission. Is it really a

multifunctional tool, or are all of the functions that support infection simply consequences of suppressing PTGS? Whatever the answer, HC-Pro is a promising bait to fish for interacting plant components and to learn more about the molecular basis of PTGS. The newly discovered function of this viral Swiss army knife will make it a tool for plant geneticists, allowing gene silencing in transgenic plants to be increased or avoided. But because exaggerated euphoria about genetransfer techniques has often been followed by unwanted gene silencing, we will probably also discover the limits to which we can manipulate gene silencing. Components such as HC-Pro are not the only factors involved, and they cannot provide a $100 \%$ effective control. Armed with the new information, it is amusing to note that the connection between gene silencing and viral resistance was first described for tobacco etch virus ${ }^{6}$, despite the fact that it carries HCPro and was the source of this protein for the new studies describing its PTGS-suppressing effect $^{1-3}$. In any case, it will be exciting to learn how plants combat this viral superweapon from their side.

Ortrun Mittelsten Scheid is at the Friedrich

Miescher Institute, PO Box 2543, CH-4002 Basel, Switzerland.

e-mail: ortrun@fmi.ch

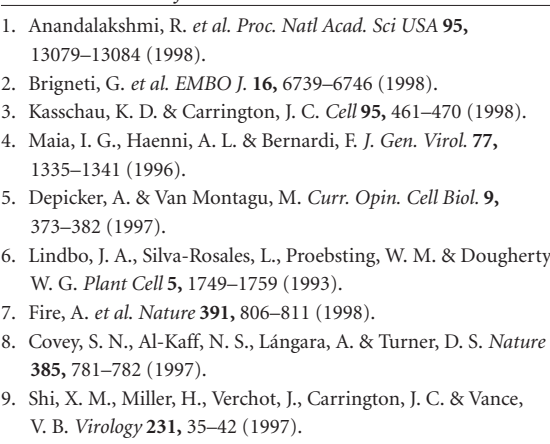

Coral reefs

\section{Recruitment in space and time}

\section{Peter F. Sale}

S ix years ago, in an address to the Ecological Society of America, Simon Levin wrote" that "the problem of pattern and scale is the central problem in ecology, unifying population biology and ecosystem science, and marrying basic and applied ecology". Interest in scale effects has since grown, and patterns or processes have been examined contemporaneously over several scales of space and time. On page 59 of this issue, Terry Hughes and colleagues ${ }^{2}$ report an important field study on patterns of recruitment and adult abundance of corals on the Great Barrier Reef.

Corals, like most reef species, have a life cycle that combines a brief, dispersive, larval phase and a sedentary (in this case immobile) adult phase. At the end of larval life, the animal must locate a suitable habitat in which to settle, metamorphose and begin its sedentary life. Ecologists term this arrival of new juveniles recruitment. For corals, suitable habitats occur in patches of reef separated by metres to thousands of kilometres of unsuitable, non-reef habitat. Transport of larval stages connects the many local populations of each species, and this interconnection drives local demography. Once marine ecologists woke up to the importance of recruitment, in the late 1970s, they found that its extent varied in both space and time $e^{3,4}$.

Hughes and colleagues have now explored variability in recruitment of corals on four spatial scales and over two successive years 


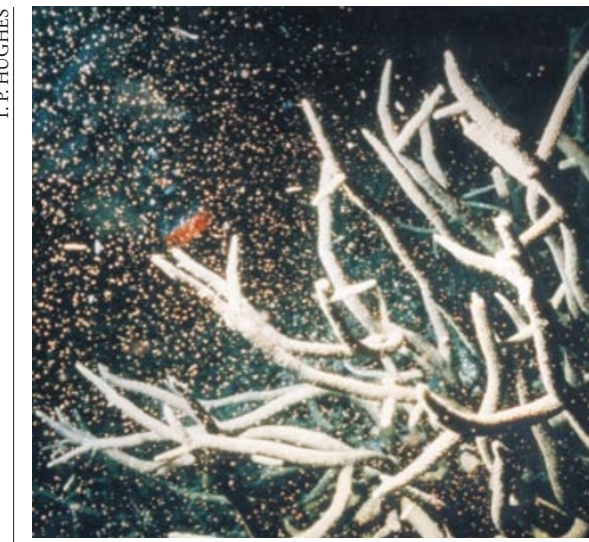

Figure 1 Corals can be divided into spawners, here seen releasing gametes during the mass spawning event, and brooders. Most of the species in the Great Barrier Reef are spawners, releasing packets of sperm or eggs for external fertilization. The resulting larvae may be pelagic for a week or more. The eggs of brooder species, however, are retained after fertilization until they are released at hatch. Hughes et al. ${ }^{2}$ took advantage of the mass spawning to study patterns of larval recruitment and adult abundances, and conclude that recruitment rates vary substantially in location and time.

(1995 and 1996), and they relate the rates of recruitment at each spatial scale to the abundance of established adults. Scales ranged from that of reef sectors $250-500 \mathrm{~km}$ apart to that between replicates only metres apart. Unglazed ceramic tiles, individually bolted to the reef platform, provided standard surfaces on which recruiting corals settled. Nearby line transects provided data on adult corals. The authors were aided by the mass spawning of corals (Fig. 1). Most species of coral on the Great Barrier Reef spawn together over just one or two nights, soon after the full Moon in November each year ${ }^{5}$. This meant that tiles deployed at each site for a brief (eight-week) period sampled the total annual recruitment to that site. Despite this cnidarian cooperativeness, Hughes and colleagues had a logistically complex task, deploying and retrieving tiles at 72 sites arrayed over $2,000 \mathrm{~km}$ of the Great Barrier Reef and, in the first year, sampling the abundances of established corals at each site.

With this study, Hughes et al. illustrate the pattern of variation in recruitment, the spatial scales at which variation is strongest, and the extent to which recruitment mirrors patterns of adult abundance. The picture, although clear, is far from simple. It is also the first such picture for corals because, although coral recruitment has been studied since the mid-1980s, all previous work has been done at single, or localized, sites.

There are three related and important take-home messages. The first is that recruitment rates vary substantially among locations on the Great Barrier Reef. This variation is greatest at scales of $250-500 \mathrm{~km}$ (sectors) and $0.5-3 \mathrm{~km}$ (sites within reefs); it is much lower at the intermediate (between-reef) scale of $10-15 \mathrm{~km}$. Recruitment rates also differ between spawner and brooder corals, and between years. The second message is that variation in recruitment does not match variation in the abundance of adults, indicating that other demographic processes also probably vary between locations. The third is that the other two messages assume that 1995 and 1996 were 'typical' years.

The third message needs to be made pointedly to those who fund ecological science. Hughes and colleagues' study is 'big' science, yet it has yielded only one small snapshot of the coral reef - this work must be repeated at other times and places. For those interested in the demography of corals, the other two messages provide a glimpse of the tangible realism that will be essential if we are to develop useful models of the dynamics of these complex open populations. These two messages are also very important for those who manage coral reefs.

Coral reefs rival rainforests in species diversity (supporting 1-9 million species), and greatly exceed them in the number of phyla (32 of 33) present $t^{6}$. As well as being important biodiversity stores, coral reefs are economically valuable to the (chiefly developing) countries that are privileged to have them. Reefs occupy less than $0.2 \%$ of the world's oceans, yet they provide $25 \%$ of the fishery catch in developing countries, plus other valuable exported products. Thriving reef environments also generate a substantial and growing revenue through tourism, and protect coastal communities from storms and marine erosion. These considerable economic and ethical values all require that the reef be managed sustainably, yet there is

growing awareness - and some alarm that, worldwide, coral reefs are being seriously degraded by human activities ${ }^{7,8}$. These include direct effects such as overfishing, and the less direct effects of pollution or, perhaps, global warming.

As a science reef management is in its infancy. We assess the condition of the reef by tedious surveys of coral cover, and similar snapshot approaches to the present state of the system. But by demonstrating that recruitment and adult population patterns are mismatched, Hughes and colleagues now show that current state need not predict current dynamics. Because a coral reef is a living system, we'd be wiser to base management on dynamics rather than state, because dynamics determine tomorrow's state.

Yes, unfortunately, the message to managers is this - your task, already difficult, just got tougher. The science that we must build to guide reef management will have many dimensions, and Hughes and colleagues have shown that the ecological dimension will not be trivial.

Peter F. Sale is in the Biology Department and the Great Lakes Institute for Environmental Research, University of Windsor, Windsor, Ontario, Canada N9B 3P4.

e-mail:sale@uwindsor.ca

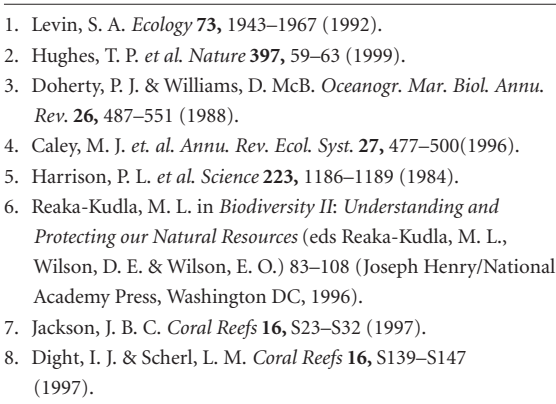
Photosynthesis Making light of a dark situation

\section{Robert Willows}

W hen the seedlings of flowering plants are grown in the dark (etiolated seedlings), they cannot make chlorophyll because the enzyme that reduces protochlorophyllide to chlorophyllide protochlorophyllide oxidoreductase (POR) - requires light. As well as collecting light for photosynthesis, chlorophylls protect the plant by dissipating excess light energy. So, when etiolated seedlings emerge from the soil into the sunlight they lack these photoprotective mechanisms. Moreover, if a seedling emerges into low light it may not be able to synthesize chlorophyll rapidly enough to take full advantage of this photosynthetic opportunity. On page 80 of this issue, Reinbothe et al. ${ }^{1}$ show that POR, which presents the plant with these dilemmas, also provides it with the solution.
The pale green colour of etiolated seedlings is due to the accumulation of protochlorophyllide complexed with a reducing agent (NADPH) and POR in the developing chloroplast (etioplast; Fig. 1, overleaf). There are two differentially regulated PORs in the etioplast ${ }^{2,3}:$ PORA is present at a high level and is negatively regulated by light; PORB is found at lower, constitutive levels. Reinbothe et al. ${ }^{1}$ have now found that, in barley, PORA and PORB form a stable complex in a ratio of 5:1, and they call this a lightharvesting POR-protochlorophyllide complex (LHPP). They also show that the PORA and PORB in these complexes are specific only for protochlorophyllide $b$ and protochlorophyllide $a$, respectively.

Reinbothe and colleagues reconstituted LHPP in vitro and found that it had different 